\title{
Sobre los nuevos modelos de negocio en las actividades radiofónicas
}

On new business models in radio activities

Aurora García González, Universidad de Vigo- auroragg@uvigo.es

Mercedes Román Portas, Universidad de Vigo-mroman@uvigo.es

\section{Resumen}

Sugieren los teóricos que estamos caminando hacia una jukebox global (Burnett, 1996) en formato digital. La cadena de valor de la música es ahora diferente tal como señala, entre otros, el italiano Richeri (2004). Quien no conoce el mercado digital, puede pensar que este nuevo cambio no supone nada respecto al modelo anterior. Sin embargo, esto no es así. Y ello ha afectado de manera particular a las actividades radiofónicas. Los modelos de negocio evolucionan adecuándose a la realidad en constante cambio y en buena parte dependiente de la innovación tecnológica y de sus usos sociales, más o menos esperados. ¿Cuál es el papel de la radio en un panorama mediático crecientemente fragmentado? El artículo aborda, por una parte, los modelos de negocio y por otra la oferta de servicio radiofónico con las nuevas tendencias en el campo de la producción, distribución y contextos particulares de contacto con los oyentes que afectan a la generación de ingresos.

\section{Palabras clave}

Radio, Nuevos negocios, Convergencia mediática, Digitalización.

\section{Abstract}

Theorists suggest that we are walking towards a global jukebox (Burnett, 1996) in digital format. The value chain of music is now different as mentioned, inter alia, the Italian Richeri (2004). Who does not know the digital market may think that this new change means nothing compared to the previous model. However, this is not so. And this has particularly affected the way radio activities. Business models evolve adapting to the changing reality and largely dependent on technological innovation and its more or less expected social uses. What is the role of radio in an increasingly fragmented media landscape? The article discusses the one hand, business models and other radio service offering with new trends in the field of production, distribution and contexts of contact with listeners affecting income generation. Keywords

Radio, New Business, Media Convergence, Digitalization.

Sumario

1. Introducción. 2. Marco teórico. 3. Revisión de la literatura. 4. Convergencia y divergencia. Causas de la pérdida de mercado publicitario. 5. Resultados: Modelos de generación de ingresos al margen de la publicidad. 6. Conclusiones. 7. Bibliografía. 


\section{Introducción}

La evolución de las tecnologías ha impulsado un mercado al que las emisoras radiofónicas convencionales estaban deseosas de incorporarse. En este panorama se han ido generando nuevos modelos de actuación basados en procesos interactivos. De acuerdo con Cebrián las cadenas emplean "esquemas de información y recursos expresivos que hacen uso del texto, el audio y el vídeo para modificarse como cibermedios". (Cebrián 2009) Estos cambios estructurales han afectado a la industria radiofónica que se enfrenta a un nuevo mercado mediático.

El entorno digital y el papel que juega el público en él abrieron las puertas a nuevas formas de transmisión de información, a través del uso y apropiación de las TIC. Con la tecnología (ordenadores, tabletas, teléfonos inteligentes y dispositivos portátiles de cualquier clase) las personas se ubican frente a una plataforma digital que contiene en su interior múltiple información, siempre disponible por el mero hecho de tener conexión a Internet.

Dada la multiplicidad de alternativas de información y entretenimiento que ofrece el mercado, la audiencia, con el concurso de la tecnología, se constituye en una comunidad con nuevas coordenadas espacio-temporales (Feijóo y García, 2016). Estas nuevas coordenadas delimitan su relación con el medio convencional y facilitan la migración del mismo al entorno digital. "Bajo este panorama, en el que tienen el mundo en la palma de su mano, el público responde a nuevas dinámicas de actuar y proceder, digitaliza cada una de sus acciones y se desenvuelve de manera adecuada en el mundo interconectado de hoy" (Orihuela, 2015).

Para hacer frente a las nuevas dinámicas de la audiencia y a los nuevos requerimientos del ecosistema comunicativo, la industria radiofónica ha necesitado volver a plantearse su funcionamiento. El cambio parece obligado debido a la concurrencia de diversas circunstancias: el mismo entorno económico, mayores disponibilidades tecnológicas, el distanciamiento o desinterés del público frente a las propuestas mediáticas convencionales, etc.

En términos de funcionamiento, predominan el liderazgo de la web y sus opciones de consumo mediático, sobre alternativas tradicionales de la radio y el transistor, lo cual plantea el interrogante de qué hacer y cómo captar la atención de los oyentes una vez superadas las fórmulas de información y entretenimiento que tuvieron su éxito en el pasado.

La audiencia actualmente utiliza más sus capacidades iconográficas, está acostumbrada a lo visual e hipertextual, es una audiencia multitasking no sólo en la escucha radiofónica como por tradición lo fue, sino que le gusta participar, trabajar en equipo, prefiere los gráficos frente a las lecturas.... "La alfabetización digital le dio cinco habilidades necesarias para el entorno digital: destrezas fotovisuales, competencias de reproducción, branching skills para buscar información, capacidad para validar y cualificar datos, e inteligencia socioemocional colectiva para actuar en las redes sociales bajo la cultura participativa" (Castellón y Jaramillo, 2009).

\section{Marco teórico}

En este contexto, la radio se ha dado cuenta de que enclaustrada en sí misma no lograría nada (Salaverría, 2014) y de que para este periodo de transición y renovación era necesario hablar no sólo con la profesión y sus actores del quehacer diario, sino que había que vincular a la audiencia en la discusión, aquellos que de una u otra manera buscan a través de la información conocer la realidad de su país y del mundo. "Los medios cambian cuando cambia la cultura de la audiencia, y aunque el cambio esté inducido por la tecnología, su naturaleza es mucho más profunda: tiene que ver con los nuevos modos en los que se produce, se distribuye y se consume la información" (Orihuela, 2015:22).

La radio utiliza su sonido convencional y se introduce en las redes sociales con una estrategia comunicativa que, por medio de una narrativa social envuelta en etiquetas y códigos, sirve para recopilar información, proponer nuevos caminos e interactuar con los oyentes en plataformas de comunicación. Estas plataformas, en palabras de Piscitelli (2014), se han convertido en la enciclopedia de consulta por excelencia por parte de la humanidad.

La relación entre la radio y los usuarios a través de la web, tal y como sostiene Cebrián (2008), se ha convertido en una red comunicativa más allá de la programación tradicional; se trata de un nuevo espacio comunicativo en que Herrera y Requejo (2012), con otros autores, plantean una distinción entre redes de flujos informativos, redes de interacción entre los propios usuarios, y redes de seguidores y del propio medio de comunicación.

Una relación en la que la radio propaga unas estrategias de marketing (Martí et. Al., 2015) para expandir su oferta e implantar la marca en el entorno on line, al tiempo que los followers la encuentran, y la siguen a ella porque les interesa clara y distintamente. Se trata de una actuación bajo las propias lógicas de las redes sociales (Orihuela, 2015), en un escenario de encuentro que permite al medio advertir "Io que está pasando en la calle, la agenda del día, el verificar el nivel de penetración e impacto de la radio con sus mensajes y el saber cuáles son esas informaciones o temáticas que parten de los ciudadanos" (Barrios, 2015).

En definitiva, lo que se empieza a comprender es que se está produciendo un cambio importante en la relación radio-audiencia que exige una comunicación permanente. Como ya se ha repetido innumerables veces, la red es una plaza pública de conversación mutua en la que confluyen contenidos, expectativas e informaciones, lo que exige del medio y por ende de sus periodistas actualizarse en esquemas y narrativas, para atender al ecosistema comunicativo multimediático de la sociedad contemporánea. Al decir de algunos autores, la radio en esta era digital expande sus horizontes y el servicio cambia, así como las competencias de los profesionales. En consecuencia, esto conduce a replantear los modos y los medios de producción, así como a impulsar la búsqueda de nuevos negocios o formas de distribución, hasta ahora poco experimentadas.

En este contexto, la publicidad juega un papel innegable tanto en los medios públicos como en internet y en los nuevos medios. Esto obedece principalmente a dos razones: la primera, y no muy alentadora, que la audiencia está prevaleciendo sobre los criterios de calidad; esto se ha sido definido como un empoderamiento de las audiencias y esas amplias audiencias son un target directo de la publicidad; y en segundo lugar porque estas transformaciones en el mercado, pueden provocar una competencia desleal. Este es el marco en el que se plantea el problema del sostenimiento de las actividades radiofónicas. 


\section{Revisión de la literatura}

Para constatar científicamente el problema que se apunta en este trabajo, se ha procedido metodológicamente de un modo sistemático. Inicialmente se ha realizado una revisión formal de los estudios y publicaciones recientes, producidos o publicados durante éste último decenio, por parte de estudiosos y expertos en el área científica de la comunicación radiofónica y de modelos de negocio de las empresas de comunicación.

Primordialmente, se constata el interés por el estudio del impacto de internet en la radio tradicional, de los cambios en el paradigma comunicativo de la radio en internet (Bonet, 2007; Cebrián, 2008, 2009; López, 2011; Sellas, 2013) y en el modelo de consumo, distribución y programación (Amoedo, Costa y Moreno, 2008; Martínez, Moreno y Amoedo, 2012; Moreno, Martínez y Amoedo, 2009). Es algo que ya va tomando cuerpo en los estudios académicos y en las actividades científicas de ámbito universitario que abordan el conocimiento sistemático de la comunicación radiofónica.

Por otra parte, el estudio del modelo de negocio y las fuentes de financiación de la radio en internet cuenta con menor acervo. Bonet (2007) con otros autores señaló, en sus primeros pasos, las líneas de cambio más relevantes en el modelo de negocio de la radio en internet. Correyero y Baladrón (2007), por su parte, después de analizar las principales cadenas de radio, constataron un desigual interés entre las emisoras a la hora de desarrollar nuevos modelos de financiación.

Herrero y Sádaba (2009) han descrito los principales agentes de webcasting en España, sus modelos de financiación y la oferta de contenidos. Puede verse en las opiniones de expertos que la situación no se refiere a un momento de crisis para volver a trabajar según el modelo anterior. La transformación es radical, como están probando los escritos e investigaciones consultadas. Es tarea común de investigadores y de directivos. Como se ha reseñado, también los empresarios buscan activamente un modelo de negocio que satisfaga el complejo entramado de intereses que afectan a productores, fabricantes de tecnología y software, distribuidores y anunciantes. Las cadenas de valor, los modelos de negocio y las funciones de las empresas están siendo cada vez más claras.

Otros estudios más recientes apuntan que los principales problemas de la industria siguen siendo la diversidad de estándares tecnológicos, la escasez de modelos de negocio, la inmadurez de la producción y la falta de apoyo de los anunciantes" (León, 2008). Es doctrina muy común en los autores que "las empresas de medios de comunicación revisan y amplían sus modelos de negocio (Campos, 2010).

Y recientemente la tesis doctoral de Carolina Díaz Espina, dirigida por Alfonso Vara, sobre nuevos modelos de negocios digitales (Universidad de Navarra, 19 septiembre de 2016), aunque se centra en actividades de prensa, subraya que desde la investigación sobre modelos de negocios en la industria de la información apenas se ha dado respuesta a este problema, en parte por la escasa uniformidad conceptual, teórica y metodológica, y en parte por una excesiva orientación hacia uno de los elementos de los modelos de negocio: los ingresos.

\section{Convergencia y divergencia. Causas de la pérdida de mercado publicitario}

El punto de partida de esta reflexión se apoya en que las actividades comunicativas se desarrollan actualmente en un espacio de convergencia y divergencia. Un entorno comunicativo que propicia un nuevo escenario marcado por la convergencia digital (Soengas, 2013). Inicialmente la convergencia fue entendida como el acercamiento de todas las formas de comunicación a través de medios electrónicos y digitales, impulsadas por la mediación de ordenadores; en este entorno, las fronteras desaparecen, y los destinatarios pueden acceder a cualquier contenido en plataformas digitales. Esta transformación trajo consigo lo que Jenkins (2006) llamó Convergence Culture.

Jenkins expone que la convergencia mediática trae consigo una preocupación latente de la industria comunicativa por brindar diversas alternativas a su información, de modo que se proporciona al usuario una serie de experiencias en cada una de las plataformas por las que circula el producto comunicativo. Domingo et. al. (2007) aluden a un concepto polisémico usado para describir, no sólo el desdibujamiento de los límites de los medios, sino el de las habilidades profesionales y los roles de los actores de los mass media. Se trata de un proceso multidimensional que para Salaverría, García y Massip (2010) afecta el ámbito tecnológico, empresarial, profesional y editorial de los medios de comunicación.

Asimismo, la definición de convergencia se ha planteado desde cuatro perspectivas:

-la de la integración de las tecnologías con la comunicación, en particular con el contenido de los medios para conquistar nuevas audiencias; -la de la apertura a nuevas alternativas de ingresos financieros;

-la de la transformación en el modo de presentar los contenidos y en consecuencia la transformación del comportamiento de las audiencias frente a ello;

-y la de la interacción social que se da en la modificación de la propuesta comunicativa del medio.

En este escenario en el que se unen los medios tradicionales y los nuevos medios y constituyen plataformas de comunicación, surgen nuevas características y propiedades. Empiezan a constituirse lo que podrían denominarse meta-medios a través de los cuales se perfilan audiencias que son participativas.

De acuerdo con Piscitelli (2014), la pérdida de cuota de mercado publicitario de las radios tradicionales obedece, entre otros factores, al surgimiento de nuevos competidores. Este hecho ha podido incidir negativamente en la migración del negocio radiofónico a la red. Al respecto, resultó particularmente llamativo el caso de los agregadores del tipo Ivoox, que, mediante la redistribución de contenidos sonoros procedentes de otras radios, lograron crear productos que revisten gran interés para anunciantes y centrales de medios. En estos casos, la mayor parte de los ingresos publicitarios son para el intermediario y no para el productor o creador de esos contenidos.

No obstante, cabe reflexionar sobre las fortalezas del modelo de financiación de la radio en internet que se podrían sintetizar en dos ideas. En primer lugar, el modelo de gratuidad de la radio puede prolongarse en la red como continuación del negocio tradicional. En segundo lugar, los estudios de audiencia muestran que los oyentes online y offline, al menos por el momento, no funcionan como sustitutos entre sí, incluso aunque 
pueden presentar características demográficas similares como han señalado varios autores. En la medida en que las audiencias de la radio sean diferentes en actividades online y offline, los anunciantes, en función del target, pueden planificar sus campañas sin que exista una competencia significativa entre ambos canales de distribución.

La digitalización ha multiplicado la competencia para la radio, el mundo digital del siglo XXI exige productos elaborados en formatos adaptables a los dispositivos portátiles del mercado y que respondan a un modelo de comunicación adecuado al nuevo ecosistema mediático. Es decir, que la radio y su propuesta sonora y digital disponen de un espacio multidireccional y simultáneo, a través de las plataformas de comunicación en la red. Un espacio en el que el medio desarrolla sus tareas informativas y que presenta al usuario la posibilidad de interactuar con el contenido del medio.

\section{Resultados: Modelos de generación de ingresos al margen de la publicidad}

Para los productores de radio y de contenidos para radio, la era digital de compartir en red ofrece oportunidades de expansión y de negocio, pero también desafíos difíciles y particularmente importantes en el sector radio. Este sector se encuentra en cambio permanente y resulta ardua la tarea de prospectiva sobre escenarios futuros.

Parece necesario localizar nuevas vías de crear riqueza mediante su valor informativo. Tal es el caso de algunas emisoras radiofónicas, que a pesar de ser absolutamente deficitarias y ausentes de lucro alguno, estando carentes de cualquier ingreso por la prestación del objeto de negocio propio suyo -el radiofónico- (como pago del oyente o cobro al anunciante), logran sin embargo mantener su rentabilidad sirviéndose de otros ingresos. Vaughan Radio en Madrid, un ejemplo muy singular, obtiene sus ingresos gracias a los cursos de idiomas que oferta y a las publicaciones educativas que factura, a pesar de estar constantemente facilitando totalmente gratis lecciones de inglés al oyente, tanto online como en antena. Los productores intentan adaptarse y sobre todo anticiparse al cambio invirtiendo en investigación y desarrollo, en experimentación e innovación de los modelos de negocio adecuados a los nuevos retos.

Se ha sostenido que estos nuevos modelos de negocio, que las empresas informativas deben adoptar, serán digitales, o no serán. El comportamiento de las audiencias se ha modificado radicalmente. Hoy todo el público quiere recibir el producto singularmente y a la carta, en su pantalla, inmediato y gratuito: nada de negocios de masas. Es una sentencia firme: "Business models are digital" (García Alonso, 2014: 735).

Sin embargo, el modelo de negocio y formas de rentabilidad de las radios on line se encuentra todavía en desarrollo. No obstante, parece que el primer paso, el intento de crear una red social extensa, goza de un relativo éxito en lo que se refiere al incremento de oyentes y de utilizadores. $Y$ es de destacar que la escala es ahora global al contrario de lo que ocurría con las radios hertzianas.

\subsection{Suscripciones}

Más allá de la publicidad, el negocio de la radio obtiene también ingresos procedentes de suscripciones_como es el caso de Last.FM o My Way. Este modelo de negocio se asienta sobre las premisas freemium. Se desarrolla permitiendo la prueba gratuita del producto a una audiencia amplia. Se trata de crear familiaridad con el producto y un hábito de consumo. El modelo es enseguida enriquecido con productos Premium (de pago) para otros segmentos de público.

El mayor beneficio de este modelo es que permite alcanzar un conjunto amplio de consumidores que constituyen la necesaria masa crítica publicitaria. La distinción de contenidos de pago tiene que ser debidamente encuadrada a través de estudios de prospección sobre la disposición máxima a pagar ("wiillingness to pay") entre distintos segmentos de consumidores. Los diferentes precios dependerán del equilibrio entre oferta y demanda. Estas suscripciones deben presentarse en forma de micro-pagos, funcionando en economía de escala y tendrán que ser de fácil acceso y uso (user-friendly).

La dimensión digital en red altera sustancialmente la economía y los modelos de negocio en red. La teoría del Long Tail de Anderson (2006), posteriormente englobada en el paquete de definiciones adyacentes a la web2.0, defiende que la tecnología global en red, Internet, revoluciona las oportunidades de negocio de venta y distribución.

Los productos con ventas relativamente bajas, si se suman, pueden ser mayores que los productos populares, llamados best sellers 0 blockbusters. La apuesta por nichos de mercado puede compensar por acumulación la escala global. Los gastos de almacenamiento son radicalmente inferiores a los soportes físicos, y posibilitan la oferta a un espacio musical amplio y ecléctico. La cuestión central del almacenamiento deja de ser un impedimento a partir del momento en que el soporte se desmaterializa para digitalizarse. De este modo las teorías económicas como las del Long Tail, que da sentido estratégico a la Distribución de Pareto: a saber, que el $80 \%$ del volumen de negocio proceden de sólo el $20 \%$ de clientes, cobran relieve en la lectura de la realidad radiofónica en red.

Este modelo de distribución abre un nuevo espacio para la difusión y distribución de productos nacionales. El mercado de oyentes se amplía por la inmensidad del espacio de hispanoparlantes (400 millones en el mundo). El Long Tail permite alimentar esperanzas de alguna rentabilidad de la producción en lengua castellana de contenidos en una economía de escala de dimensión global.

\subsection{Asociaciones y alianzas}

En las nuevas radios on line, más allá de los modelos de ingresos procedentes de dividendos publicitarios y suscripciones, existe todavía la posibilidad de ventas de una forma más o menos directa. Los paralelismos entre una radio y una tienda de discos on line están patentes en el concepto de la mayoría de las radios on line con asociaciones con minoristas en la red. La escucha de un determinado fragmento puede potenciar su adquisición, de forma rápida en búsquedas y consumos cada vez más bajo la premisa de "a la carta" o unbundled.

La asociación entre servicios on line en forma de mashup (en términos de desarrollo web, una mashup es una forma de integración y reutilización. Se da cuando una aplicación web es usada o llamada desde otra aplicación, con el fin de reutilizar su contenido y/o funcionalidad) es una tendencia creciente. La comunicación de servicios con vistas a conseguir una vertiente más social se puede apreciar sobre todo a través de la 
integración con Facebook (un posible ejemplo de la intensificación de la tendencia Cloud radio). El potencial de interacción social procede de la fuerza de la recomendación por pares que es la premisa por ejemplo de Last.Fm.

Estas asociaciones pueden jugar un gran papel en la radio, con el fin de capitalizar consumos de música on line o conciertos en vivo. Y pueden establecerse entre oyentes de radio y lectores de periódicos como hizo en su día MyWay con el diario "i" en Portugal o $A B C$ con Punto radio en España.

Estas alianzas son la nota dominante en las empresas multimedia que se rigen por lógicas de integración; son asociaciones con empresas contiguas no sólo multimedia sino de nuevas tecnologías. Por ejemplo, en Last.fm aparecen pequeñas publicidades textuales en Google, y también pequeños banners comerciales y links con la tienda iTunes o Amazon para la compra de música, de un álbum o la discografía completa del artista que se oye en cada momento.

Si la asociación con Amazon es una estrategia interesante de sinergia de Last.Fm, que se entiende fácilmente, conviene prestar atención con detalle a la eficacia del servicio de publicidad introducido por parte de Google. El servicio ofrecido por la radio Last.Fm es gratuito, aunque incluye la posibilidad de suscribir una cuenta de pago que, entre otros beneficios, ofrece la retirada de la publicidad.

Además de estas alianzas con servicios sociales de venta de música on line o publicidad hay también asociaciones con editoras discográficas. Las radios, sobre todo aquellas de piloto automático, quieren ampliar su oferta. Estas asociaciones permiten ofrecer un abanico musical cada vez más amplio y diversificado, que hace posible una experiencia de radio más rica para el consumidor. Por un lado, le pone al alcance un ámbito de descubrimientos y socialización musical mayor y por otro le permite una mejor adecuación entre oferta y demanda de gustos musicales.

Por parte de las empresas editoras de música el interés también es obvio. Estas coaliciones de intereses demuestran la percepción por parte de las discográficas de la importancia de estos servicios de distribución, difusión y promoción de contenidos a escala global y la creación de nuevos públicos y modelos de negocio. Por ejemplo, Last.Fm firmó acuerdos con múltiples editoras independientes y con la multinacional Sony BMG y la Warner Music aunque desistieran posteriormente. MyWay firmó acuerdos con Sony Music y Universal.

Asimismo, MyWay estableció una asociación con la agencia de noticias Reuters con la intención de publicar noticias en formato vídeo haciéndose MyWay responsable de la edición y locución de los vídeos. Y Last-Fm se alió con Microsoft a finales de 2009 en algunos países en que los miembros de Xbox Live Gold podían acceder a sus contenidos de pago.

Son algunos de los ejemplos que permiten entender la evolución natural de la radio analógica y su modelo empresarial a la radio digital y su integración en la red, con sus diferencias de procedimiento para acometer nuevos negocios. En un mundo global no cabe apoltronarse en situaciones previas.

\section{Conclusiones}

Los nuevos sistemas de difusión sustituyen en parte a los tradicionales; pero también potencian recursos antiguos, cambiando sus aspectos de continuidad. La radio es un estilo comunicacional muy adaptable al medio tecnológico (analógico o digital) con el que se opera. Esta característica es un atributo que favorece su permanencia en un terreno muy diferente de aquél en que nació.

- Hay que tener en consideración otro factor, de naturaleza sociológica, que es la emergencia del individuo conectado. El espacio comunicativo es hoy mucho más denso y los contactos entre actores sociales están potenciados por herramientas digitales que, en simultáneo, contraen el tiempo y el espacio.

- La proliferación de ofertas de contenidos ha producido una disminución de la atención

que revierte en contra de los contenidos radiofónicos. Como vía para minimizar los esfuerzos de búsqueda los usuarios cuentan cada vez más con redes personales que les ayudan a seleccionar y evaluar los estímulos que reciben. El nuevo paradigma digital ha transformado los conceptos de espacio y tiempo.

- Las funciones sociales la radio y de la webradio recuerdan la presencia constante de un espacio público, colectivo, que existe (y del que se forma parte) también, aunque no se identifique con un lugar físico exclusivamente y aunque se frecuente sólo eventualmente.

- La nueva radio tiene poco que ver con el medio tradicional. El fenómeno de la participación supone una ruptura cognitiva y cultural y se encuentra estrechamente ligada a la naturaleza de la relación entre los medios y la sociedad.

- La participación directa de la audiencia en la programación radiofónica habilitada ha propiciado el intercambio profesional entre los roles de emisor y de receptor y hecho posible que los oyentes intervengan en el contenido de las emisiones. En la comprensión del alcance que tiene la participación directa de la audiencia en las actuales ofertas programáticas resulta decisiva la influencia de los cambios estructurales y narrativos que se han sucedido en la radio en los últimos 20 años, como consecuencia del impacto de las nuevas tecnologías.

- Existe, con todo, todavía una sobrevaloración del campo programático sobre las posibilidades que la web ofrece a su dimensión social, relativas a la capacidad de cualquier fórmula participativa para poner en contacto profesionales y audiencias y contribuir a la configuración de la radio como actor social.

\section{Bibliografía}

Anderson, Ch. (2006) The Long Tail. USA. Hyperion

Amoedo, A., Martínez Costa, Ma P. y Moreno, E. (2008) "An analysis of the communication strategies of spanish commercial music networks on the web: los40.com, los40principales.com, cadena100.es, europafm.es and kissfm.es" en The Radio Journal. International Studies in Broadcast \& Audio Media 6 (1), 5-20

Barrios, A. (2015) "La radio colombiana frente al reto digital" en Comunicación y medios $\mathrm{N}^{0} 31$

Bonet, M. (2007) "Nuevos cambios para la radio. Un proceso productivo digital para un negocio analógico". En Telos № 73 
Burnett, R. (1996) The Global Jukebox: The International Music Industry. Londres. Routledge. Castellón y Jaramillo (2009) "Nativos digitales en los entornos universitarios". Textual \& Visual Media 6, 2013 (177-196)

Cea, M.N. (2009) "Modelo de negocio de la empresa periodística en Internet: el caso de Prisa" en Revista Latina de comunicación social. 64 (938950

Cebrián, M. (2008) La radio en Internet . Buenos Aires. La Crujía

Cebrián, M. (2009) "Comunicación interactiva en los medios" en Comunicar 17: 33, 15-24.

Cebrián, M. (2009) "Nuevas formas de comunicación: cibermedios y medios móviles". Comunicar 17.

Correyero, B. y Baladrón, A. (2007) "El podcasting en los medios de comunicación españoles". Libro de Actas del $8^{\circ}$ Congreso de Periodismo Digital. 154-169.

Domingo et. Al. (2007) "Four dimensions of Journalistic convergence. A preliminary approach to current media trends at Spain". Internatioanl Congress on Online Journalism. Austin Texas.

Feijóo Fernández, B. Y García González, A. (2016) "Mayores universitarios y usos de Facebook". Comunicación y Hombre 12 (305-318)

Franquet, R. (1999) "El Dab un repte technologic". Quaderns del CAC 4 (26-29)

García Alonso, P. (2014) "Nuevos modelos de negocio" en Historia y Comunicación Social 735 Vol. 19.N Esp.Marzo (729-741)

Herrera y Requejo (2012) "10 Good Practices for News Organizations Using Twitter", en Journal of Applied Journalism and Media Studies, vol. 1.

Herrero, M.; Sádaba, C. (2009). "El mercado de webcasting en España: fortalezas y desafíos". Comunicación presentada en el IX Congreso Iberoamericano de Comunicación.

Jaramillo (2011) Redes sociales para todos. Su negocio en la web 2.0. Bogotá. Ediciones B

Jenkins, H. (2006) Convergence Culture, where old and new media collide. USA. New York University Press

Jódar (2010) La era digital: nuevos medios, usuarios y nuevos profesionales. Razón y Palabra (71).

León Anguiano, B. (2008): "La visión de los productores sobre la televisión interactiva: el final de la utopía”, en Comunicación y Socidad, vol. XXI, NN1 / 2008, http://www.unav.es/fcom/comunicacionysociedad/es/articulo. php?art_id=41 [22-09-2013]

López, N. (2011) La radio se transforma: nuevas tecnologías, nuevos hábitos de consumo y nuevos perfiles para el medio más cercano. En Ortiz, M.A. y López, N. (eds) Radio 3.0 Una nueva radio, para una nueva era: la democratización de los contenidos (pp. 15-40) Editorial Fragua. Madrid Martí et. Al. (2015) La radio modelo de negocio en transición: estrategias de oferta y de comercialización en el contexto digital en Quaderns del CAC XVIII (41), (13-22)

Martín, R.F. y Fernández, J. (2012) La digitalización como eje de transformación de las agencias de medios españolas. Pensar la publicidad. 6 (2). 427-445

Martínez (2009)

Martínez Costa y Amoedo (2012) "La radio generalista en la red: un nuevo modelo para la radio tradicional". En: Anagramas, vol.10, n 20 pp. $165-$ 180, Medellín Disponible en: http://www.scielo.org.co/pdf/angr/v10n20/v10n20a12.pdf [13 de enero de 2014].

Orihuela (2015) Los medios después de internet (Editorial UOC, 2015)

Pérez y Gallego (2010)

Piscitelli (2014) "Internet la imprenta del sigloXXI" en VV.AA. Contra el determinismo tecnológico. Cibercultura

Richeri, G. (2004) “La televisión digital terrestre en Europa. Un camino plagado de incertidumbres". Telos, Segunda Época, n58

Salaverría, R. (2014) Tendencias del periodismo mundial. Periodismo en 2014: balance y tendencias en Cuadernos de periodistas: revista de la Asociación de la Prensa de Madrid. № 29, 9-22

Salaverría R. (2009) Los medios de comunicación ante la convergencia digital. En Actas del I Congreso Internacional de Ciberperiodismo y Web 2.0 (CD-Rom) Bilbao, 11-13 de noviembre. Servicio editorial de la UPV-2014

Salaverría, R; García, J.A. y Massip, P (2008) "Concepto de convergencia periodística" en X López García y X. Pereira (eds), Convergencia digital. Reconfiguración de los medios de comunicación en España. Santiago. Servicio de Publicaciones de la Universidad de Santiago (41-64)

Scolari, C. (2004) Hacer clic. Hacia una sociosemiótica de las interacciones digitales. Barcelona Gedisa.

Sellas, T. (2013) "Radio y redes sociales: los magazines matinales den Twitter" en Quaderns del CAC XVI (39). 25-36

Soengas, X. (2013) Retos de la radio en los escenarios de convergencia digital. En AdComunica Revista de Estrategias, Tendencias e Innovación en Comunicación (5) 23-26.

\section{Cómo citar este artículo en bibliografías - How to cite this article in bibliographies / references:}

GARCÍA-GONZÁLEZ, A. (2017): Sobre los nuevos modelos de negocio en las actividades radiofónicas”. En Revista de la Asociación Española de Investigación de la Comunicación, vol. 4, número 7, pp. 40-45. 